\title{
Identification of Candidate Genes for Self-Compatibility in Perennial Ryegrass (Lolium perenne L.)
}

\author{
Claudio Cropano ${ }^{1,2+}$, Chloé Manzanares ${ }^{1}$, Steven Yates ${ }^{1}$, Dario Copetti 1,3t, \\ Javier Do Canto ${ }^{4}$, Thomas Lübberstedt ${ }^{5}$, Michael Koch ${ }^{2}$ and Bruno Studer ${ }^{1 *}$ \\ ${ }^{1}$ Molecular Plant Breeding, Institute of Agricultural Sciences, ETH Zurich, Zurich, Switzerland, ${ }^{2}$ Deutsche Saatveredelung \\ AG, Lippstadt, Germany, ${ }^{3}$ Department of Evolutionary Biology and Environmental Studies, University of Zurich, Zurich, \\ Switzerland, ${ }^{4}$ Instituto Nacional de Investigación Agropecuaria, Tacuarembó, Uruguay, ${ }^{5}$ Department of Agronomy, lowa \\ State University, Ames, IA, United States
}

OPEN ACCESS

Edited by:

Petr Smýkal,

Palacký University, Czechia

Reviewed by:

Jonathan Elias Maldonado,

Pontificia Universidad Católica de

Chile, Chile

Toshihiko Yamada,

Hokkaido University, Japan

*Correspondence:

Bruno Studer

bruno.studer@usys.ethz.ch

tPresent address:

Claudio Cropano,

Puregene AG, Zeiningen, Switzerland

Dario Copetti,

Arizona Genomics Institute, University of Arizona, Tucson, AZ, United States

Specialty section: This article was submitted to Plant Breeding,

a section of the journal

Frontiers in Plant Science

Received: 10 May 2021 Accepted: 30 August 2021 Published: 15 October 2021

Citation:

Cropano C, Manzanares C, Yates $S$, Copetti D, Do Canto J, Lübberstedt T, Koch M and Studer B (2021)

Identification of Candidate Genes for Self-Compatibility in Perennial Ryegrass (Lolium perenne L.).

Front. Plant Sci. 12:707901. doi: 10.3389/fpls.2021.707901
Self-incompatibility (SI) is a genetic mechanism preventing self-pollination in $\sim 40 \%$ of plant species. Two multiallelic loci, called $S$ and $Z$, control the gametophytic SI system of the grass family (Poaceae), which contains all major forage grasses. Loci independent from $S$ and $Z$ have been reported to disrupt $S I$ and lead to self-compatibility (SC). A locus causing SC in perennial ryegrass (Lolium perenne L.) was previously mapped on linkage group (LG) 5 in an $F_{2}$ population segregating for SC. Using a subset of the same population ( $n=68$ ), we first performed low-resolution quantitative trait locus (QTL) mapping to exclude the presence of additional, previously undetected contributors to SC. The previously reported QTL on LG 5 explained $38.4 \%$ of the phenotypic variation, and no significant contribution from other genomic regions was found. This was verified by the presence of significantly distorted markers in the region overlapping with the QTL. Second, we fine mapped the QTL to 0.26 centimorgan (CM) using additional 2,056 plants and 23 novel sequence-based markers. Using Italian ryegrass (Lolium multiflorum Lam.) genome assembly as a reference, the markers flanking SC were estimated to span a $\sim 3 \mathrm{Mb}$ region encoding for 57 predicted genes. Among these, seven genes were proposed as relevant candidate genes based on their annotation and function described in previous studies. Our study is a step forward to identify SC genes in forage grasses and provides diagnostic markers for marker-assisted introgression of SC into elite germplasm.

Keywords: self-incompatibility (SI), self-compatibility (SC), perennial ryegrass (Lolium perenne L.), quantitative trait locus (QTL), segregation distortion, fine mapping, candidate genes

\section{INTRODUCTION}

Several species of the grass family (Poaceae) are economically valuable forage crops and represent a fundamental component of our grasslands. Most forage grasses are obligate outcrosses due to a gametophytic self-incompatibility (SI) system that prevents inbreeding (Cornish et al., 1979). Unlike most self-incompatible species, SI in grasses is controlled by two multiallelic loci, called $S$ and $Z$. Their existence is known since more than half a century (Hayman, 1956; Lundqvist, 1956), but, despite several mapping efforts (Voylokov et al., 1998; Thorogood and Armstead, 2002; Bian et al., 2004; Hackauf and Wehling, 2005; Kakeda et al., 2008; Shinozuka et al., 2010), the identity of the genes involved is unknown. To date, two genes encoding for proteins with a DUF247 domain 
on linkage groups (LGs) 1 and 2 are the most promising candidates for $S$ and $Z$, respectively, in perennial ryegrass (Lolium perenne L.) (Shinozuka et al., 2010; Manzanares et al., 2016a).

There is a continued need to accelerate breeding in forage grasses for traits, such as biomass yield and quality, seed yield, and disease resistance (Capstaff and Miller, 2018). However, the genetic gain achieved in self-incompatible grasses with population breeding strategies has lagged behind compared to inbred crops, such as maize (Zea mays L.), wheat (Triticum aestivum L.), and rice (Oryza sativa L.) (Laidig et al., 2014). This is partially caused by the inability to develop $F_{1}$-hybrid cultivars and exploit the hybrid vigor that occurs when two genetically distant (and usually highly homozygous) parents are crossed. Thus, the possibility to overcome SI and exploit selfcompatibility (SC) to transition forage grasses to an inbred linebased breeding system is gaining traction (Do Canto et al., 2016; Herridge et al., 2020). Inbreeding by self-pollination fixes favorable genetic variants and purges deleterious alleles with high efficiency, resulting in highly homozygous inbred lines (Jansky et al., 2016). Inbred lines belonging to different heterotic groups can be crossed to systematically assemble desirable combinations of genes and maximize heterosis.

Several routes to SC have been described in grasses: SC can arise from mutations at $S$ and/or $Z$ that disrupt the initial self/non-self recognition between pollen and stigma (Do Canto et al., 2016). Mutations in loci non-allelic to $S$ and $Z$ can also lead to SC, by interrupting the downstream cascade triggered by the initial self recognition (Do Canto et al., 2016). Such putative mutations have been found in rye (Secale cereale L.) and perennial ryegrass (Voylokov et al., 1993; Egorova et al., 2000; Thorogood et al., 2005; Arias-Aguirre et al., 2013; Do Canto et al., 2018; Slatter et al., 2020). A detailed molecular understanding of how SC arises in SI grasses is lacking, but several studies have acknowledged that calcium $\left(\mathrm{Ca}^{2+}\right)$ signaling plays an essential role in the recognition and/or inhibition of self-pollen (Yang et al., 2009; Chen et al., 2019). In fact, SI in grasses can be partially overcome by treating self-pollinated stigmas with chemical reagents affecting $\mathrm{Ca}^{2+}$ channeling across cell membranes (Klaas et al., 2011).

Two consecutive studies provided evidence on the location of an SC source segregating in an $\mathrm{F}_{2}$ population of perennial ryegrass. Using in vitro pollinations, Arias-Aguirre et al. (2013) observed a 1:1 segregation into two phenotypic SC classes: plants showing a $50 \%$ pollen compatibility where half of the self-pollen germinated and grew a pollen tube upon contact with the stigma, and $100 \%$ SC where all self-pollen showed a compatible reaction. It was concluded that SC was caused by a putative mutation in a major gametophytically-acting gene mapped to a 16-centimorgan (cM) locus on LG 5. Do Canto et al. (2018) fine mapped the position of the locus to a 1.6-cM region. Despite providing evidence of one major locus explaining the SC variation, these two studies did not exclude the presence of additional major effects on other LGs. In addition, the genetic region co-segregating with SC in Do Canto et al. (2018) was inferred based on genome sequence information from rice and Brachypodium (Brachypodium distachyon (L.) P. Beauv.) and is still considerably large, making it difficult to identify putative candidate genes for functional validation.

Building on these previous studies, we aimed at providing additional evidence for the quantitative trait locus (QTL) on LG 5 being the sole cause for SC variation in this population. An additional objective was to increase the genetic mapping resolution achieved by Do Canto et al. (2018) substantially, to facilitate transitioning from a genetic to a physical map in ryegrass, and to select and prioritize candidate genes in the target region. A better understanding of the genetic causes of SC can help biologists to determine the pathways involved in the SI system of grasses. In addition, it paves the way for shifting the breeding of perennial grasses from population-based to pure line-based $\mathrm{F}_{1}$ hybrid strategies.

\section{MATERIALS AND METHODS}

\section{Plant Material}

This study was entirely based on the perennial ryegrass $F_{2}$ population segregating for SC described in Arias-Aguirre et al. (2013) and Do Canto et al. (2018). The population was obtained by self-pollinating a single $F_{1}$ individual originating from the initial cross between a self-compatible genotype (selfed for five generations) and a self-incompatible individual of the $\operatorname{VrnA}$ mapping population (Jensen et al., 2005). A subset of 74 individuals of the population used in Do Canto et al. (2018) was used as starting material for linkage map construction, low-resolution QTL mapping, and the analysis of segregation distortion. A larger set of 2,056 individuals, in addition to the 1,248 reported in Do Canto et al. (2018), was used for the fine mapping.

\section{DNA Extraction}

All genomic DNA used in this study was extracted from powdered frozen leaf tissue using the Mag-Bind ${ }^{\circledR}$ Plant DNA DS 96 Kit (Omega Bio-tek, Inc. Norcross, GA, USA) on a 96-well plate KingFisher Flex Purification System (Thermo Fischer Scientific, Waltham, MA, USA) following the recommendations of the manufacturer. Genomic DNA was visualized on a $1 \%$ agarose gel and quantified with a NanoDrop 8,000 spectrophotometer (Thermo Fisher Scientific, Waltham, MA, USA).

\section{QTL Mapping and Analysis of Segregation Distortion}

\section{Phenotyping for Self-Compatibility}

The SC phenotypic data of the $74 \mathrm{~F}_{2}$ individuals were obtained from the in vitro pollination assay data reported in Do Canto et al. (2018). Briefly, the number of compatible pollen grains, usually translucent, and with bright pollen tubes growing toward the style, was counted on at least 10 stigmas per plant. The data for each plant consisted of the overall mean percentage of self-compatible pollen, using the stigmas as subsamples. Chi-square goodness of fit test was performed to test if the phenotypic variation deviated from the hypothesis of SC being under the control of a single a gametophytically-acting gene. In that case, only heterozygote and homozygote plants for the SC 
allele (corresponding to the 50 and $100 \%$ phenotypic class) are expected in a 1:1 ratio in the $\mathrm{F}_{2}$.

\section{Genotyping-by-Sequencing, Data Processing, and DNA Variant Calling}

A genotyping-by-sequencing (GBS) library was prepared following the protocol reported in Begheyn et al. (2018) starting from genomic DNA of 74 individuals of the $\mathrm{F}_{2}$ population and sequenced using 125 bp single-end reads on an Illumina HiSeq 2,500 platform at the Functional Genomics Center Zurich (FGCZ), Zurich, Switzerland. Sequencing reads were demultiplexed using Sabre (https://github.com/najoshi/sabre), allowing no mismatches. After demultiplexing, all adapters and barcodes were removed from the reads, which were then trimmed to $100 \mathrm{bp}$ to remove barcodes and poor-quality sequences $(\mathrm{Q}<30)$. In absence of a chromosome-scale assembly available for perennial ryegrass, variant calling was performed with the GBSmode pipeline (Yates and Studer, 2021), using as a reference the genome assembly of Italian ryegrass (Lolium multiflorum Lam.), a self-incompatible species showing high synteny and relatedness to perennial ryegrass (Copetti et al., 2021).

\section{Genetic Linkage Map Construction}

A genetic linkage map was constructed using $\mathrm{R}$ (version 4.0.0) ( $\mathrm{R}$ Core Team, 2020) with the R/qtl package (Broman et al., 2003). Single nucleotide polymorphisms (SNPs) resulting from the GBS variant calls were filtered for a 5\% minor allele frequency (MAF) and $<40 \%$ missing values. Individuals showing $<40 \%$ missing genotype calls were also filtered. In addition, pairs of individuals with more than $90 \%$ matching genotypes were identified and one was removed. As a result, 473 highquality SNPs and 68 individuals were kept for the construction of the genetic map. Markers were grouped in LGs with the formLinkageGroups function with a maximum recombination rate of 0.35 and minimum $-\log _{10}$ ( $p$-value) logarithm of odds (LOD) threshold of 6 . The initial marker order and genetic distances were established using the Kosambi mapping function $[d=(1 / 4) \ln (1+2 r / 1-2 r)]$, where $d$ is the mapping distance and $r$ is the recombination frequency (Kosambi, 1943). Singletons and double recombinations inflating the map size were identified and corrected using a graphical genotyping approach (Young and Tanksley, 1989). All singletons not followed by at least four markers of the same haplotype were turned into missing values and marker order and genetic distances were re-calculated. This process was repeated until all singletons and double recombinations within a window of four markers were removed. LG numbers were assigned based on the physical position of their markers on the Italian ryegrass assembly (Copetti et al., 2021).

\section{Quantitative Trait Locus Mapping and Analysis of Segregation Distortion}

The R package R/qtl was used to perform QTL mapping (Broman et al., 2003). Missing values in the genotype table were filled with the fill.geno function, and QTL mapping was carried out using the mqmscan function, which scans the genome with a multiple QTL model based on haplotype dominance and considers additive effects. After running 1,000 permutations with an assumed genotyping error rate of 0.05 , an LOD of 2.81 was set as the QTL significance threshold. Segregation distortion was calculated using a chi-square goodness of fit test to identify markers that significantly differed from the 1:2:1 expected segregation. A Bonferroni-corrected significance threshold was set at an LOD of 3.97 .

\section{Fine Mapping}

\section{Marker Development for Fine Mapping}

To detect novel DNA sequence polymorphisms for marker development, $10 \mathrm{ng}$ of genomic DNA from 50 individuals (25 individuals homozygous for both flanking markers G05_065 and G06_096 and 25 individuals heterozygous for both markers) belonging to the collection of $2,056 \mathrm{~F}_{2}$ individuals was pooled and sequenced at around $0.5 \times$ coverage in $2 \times 150 \mathrm{bp}$ mode on an S4 flow cell lane of an Illumina Novaseq instrument at FGCZ. The paired-end reads were mapped using bowtie2 (v3.5.1) (Langmead and Salzberg, 2012) to an Italian ryegrass reference assembly (Copetti et al., 2021). The alignments were converted to BAM format with SAMtools (v1.10) (Li et al., 2009). A BAM file containing reads that aligned on the scaffolds delimited by the markers flanking the SC locus in the study by Do Canto et al. (2018) (G05_065 and G05_095, Studer et al., 2008) was imported into the Integrative Genomics Viewer software (Robinson et al., 2011), and polymorphisms were visually inspected. To develop PCR-based markers, 50-120 bp of consensus sequence spanning a single polymorphism showing the expected $50 \%$ frequency was extracted and used as a template for primer design. Primers were designed with Primer-BLAST (Ye et al., 2012) using $59-61^{\circ} \mathrm{C}$ as optimal melting temperature. Primer sets with the lowest selfand self-3'-complementarity (self-binding affinity) were chosen and tested for specificity. Similarly, BAM files of 25 genotypes with a $50 \%$ SC phenotype and 25 genotypes with a $100 \%$ SC phenotype used for GBS were also used as a template for PCRbased marker development.

\section{Large-Scale Genotyping Based on High-Resolution Melting Analysis}

Genotyping was performed with high-resolution melting analysis (HRMA) on a LightCycler ${ }^{\circledR} 480$ System (Roche, Basel, Switzerland) using the same conditions as Do Canto et al. (2018). To identify additional individuals carrying recombination, $2,056 \mathrm{~F}_{2}$ plants were genotyped with the markers G05_065 and G06_096 (Studer et al., 2008). The recombinants were further genotyped with 23 newly validated polymorphisms (Supplementary Table 1). The genotypes of G05_065 and G06_096 and the new markers were combined into a local linkage map using the OneMap software (Margarido et al., 2007). Among the new markers, seven non-co-segregating markers were used to obtain marker order and genetic distances by estimating the multipoint likelihood of all the possible orders using the compare function. The Kosambi mapping function was used for the estimation of mapping distances. The markers within the SC locus were positioned by BLASTN on the Italian ryegrass genome assembly to obtain physical distances and extract information of the annotated protein-coding genes. 

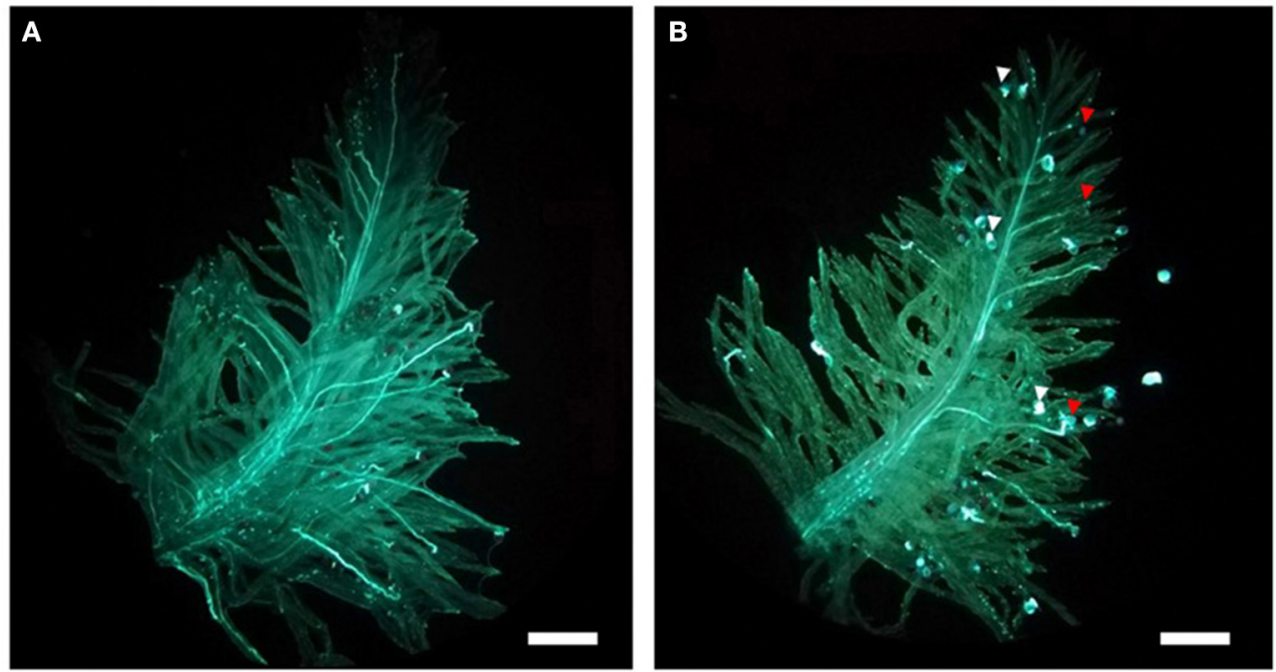

FIGURE 1 | The two self-compatibility (SC) phenotypic classes segregating in the $\mathrm{F}_{2}$ population as visible in an in vitro pollination assay. (A) In genotypes showing $100 \%$ SC, all pollen grains form a pollen tube that is able to elongate within the stigma reaching the ovary. (B) In genotypes showing $50 \%$ SC, only half of the pollen grains penetrate the stigma and elongate a pollen tube (indicated by the red arrows). For the remaining half, pollen tube growth is aborted upon contact with the stigma and followed by rapid deposition of callose at the pollen tube tip (white arrows). Bars: $400 \mu \mathrm{m}$.

\section{Phenotyping of the Recombinants for Self-Compatibility}

The individuals showing recombination between G05_065 and G06_096 were phenotyped using in vitro pollinations. Eight to ten mature virgin pistils were dissected from florets between 08:00 a.m. and 09:00 a.m. and placed on a petri dish containing a medium consisting of $2 \%$ agarose, $10 \%$ sucrose, and 100 -ppm boric acid. From the same plants, four to five inflorescences with flowering florets were enclosed in paper bags prior to anthesis to collect pollen. Around noon, the paper bags were shaken, and the pollen that fell at the bottom of the bag was sprinkled on the stigmas in the Petri dishes. After a minimum of $3 \mathrm{~h}$, the pollinated stigmas were detached from the ovary with a razor blade, moved to a microscope slide, and submerged in a few drops of a staining solution containing $0.2 \%$ aniline blue and $2 \% \mathrm{~K}_{3} \mathrm{PO}_{4}$ (Martin, 1959). After covering with a cover slide, the samples were allowed to stain overnight. Pollen growth was detected using a Leica DM12000M UV light microscope (Leica Microsystems, Wetzlar, Germany). Plants were classified as either of the two SC phenotype classes segregating in the population $(100 \%$ or $50 \%$ SC) according to the proportion of pollen grains showing a compatible reaction (Figure 1). In 100\% SC plants, all pollen grains are translucent and displayed a clear pollen tube growing toward the style. In the $50 \%$ SC plants, nearly half of the pollen grains showed a short, thickened, and bright blue pollen tube, with arrested growth upon contact with the stigma.

\section{Identification of Candidate Genes in the Genomic Region Co-segregating With Self-Compatibility}

MCScanX (Wang et al., 2012) was used to detect collinearity of the genes extracted from Italian ryegrass predicted in the genomic region co-segregating with $\mathrm{SC}$ with perennial ryegrass
(Byrne et al., 2015), barley (Hordeum vulgare L.) (Mascher et al., 2017), rice (Ouyang et al., 2007), and Arabidopsis thaliana (The Arabidopsis Genome Initiative, 2000) genome references by aligning their proteomes with BLASTP (Camacho et al., 2009) at default parameters. BLASTP hits were retained, if they had an e-value lower than $1 \mathrm{e}^{-5}$ and if they showed at least $80 \%$ (with perennial ryegrass) and 60\% (with rice and barley) similarity to an Italian ryegrass gene. For Arabidopsis thaliana, the gene with the highest similarity was chosen. Candidate genes were prioritized primarily based on functional annotation. Additional insights on their involvement in SC/SI were based on organ-specific gene expression data available on Genevestigator $^{\circledR}$ (Zimmermann et al., 2005) for Arabidopsis thaliana, barley, and rice orthologs. The pollen and stigma transcriptome data reported in Byrne et al. (2015) were used to assess if the perennial ryegrass genes present in the SC locus were differentially expressed during compatible/ incompatible reactions.

\section{RESULTS}

\section{Quantitative Trait Locus Mapping Phenotypic Segregation}

Analysis of the in vitro pollination data for the 74 individuals from Do Canto et al. (2018) grouped the plants in two clear phenotypic classes (Figure 2A). The first class consisted of 43 plants with mean compatibility of $90.5 \%$ (SD 11.0\%). Thirtyone plants were assigned to the second phenotypic class with a compatibility mean of $49 \%$ (SD 11.6\%). Chi-square test for goodness of fit showed no significant difference between observed and expected phenotypes of a monogenic trait under gametophytic control segregating in a $1: 1$ ratio in an $F_{2}$ generation $(p=0.128)$. 


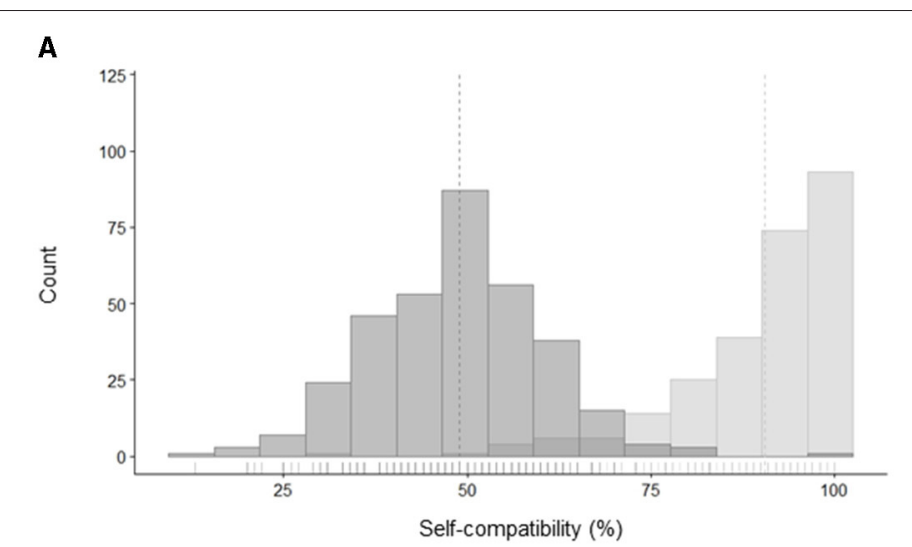

C

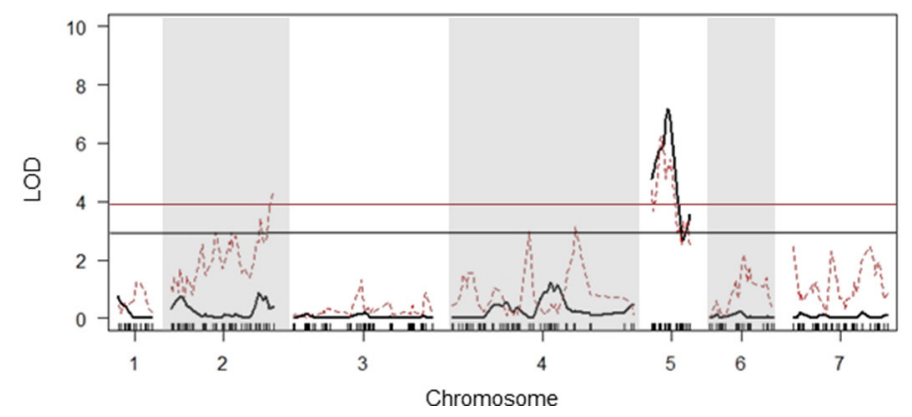

B

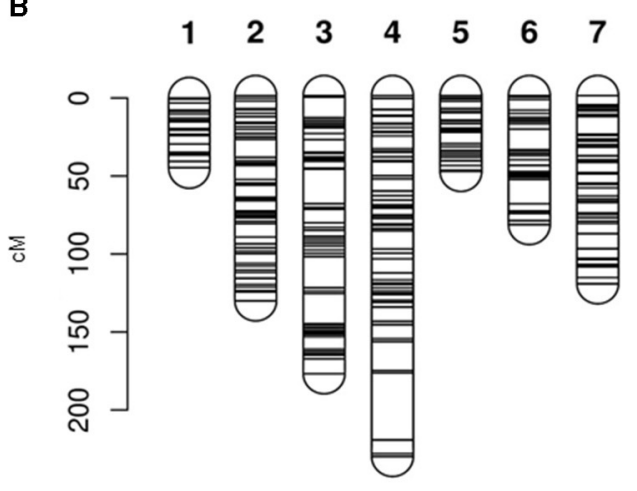

D

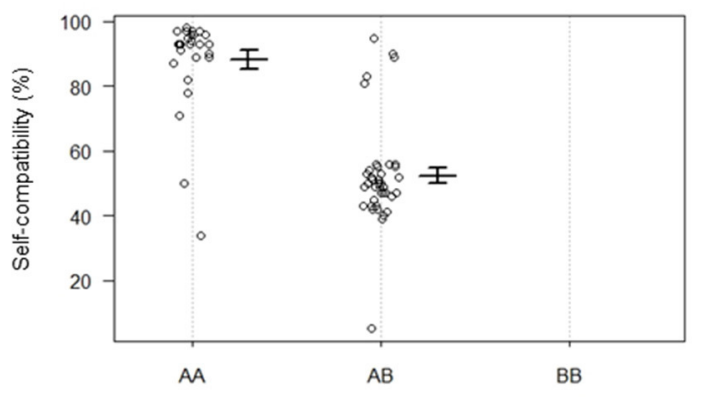

FIGURE 2 | Quantitative trait locus (QTL) analysis for self-compatibility (SC). (A) The bi-modal phenotypic segregation for the SC phenotype of the 74 individuals was used for the generation of the linkage map, QTL analysis, and segregation distortion analysis. Data distributions and means (indicated by the vertical dotted lines) displayed in dark gray for individuals with a $50 \%$ SC phenotype and light gray for the $100 \%$ self-compatible individuals. Small solid vertical lines above the horizontal axis indicate single data points. (B) Genetic linkage map displaying the distribution of the 473 markers across the seven linkage groups of perennial ryegrass used for the QTL analysis. (C) QTL analysis for SC (black line) and analysis of segregation distortion (red dotted line). Significance thresholds are indicated by the black and red horizontal lines for the QTL analysis and segregation distortion analysis, respectively. (D) Marker effect plot for the most significant marker. AA represents the homozygous genotype for the SC phenotype, while AB and BB represent the heterozygous and homozygous genotypes for the self-incompatible phenotype, respectively. Plots show the mean and SD for the SC phenotype.

\section{Genetic Linkage and QTL Mapping}

Out of 10,487 SNPs resulting from the GBS variant calling, 473 passed the stringent quality filtering and were used for linkage map construction and QTL mapping. The distribution of the markers across the seven LGs ranged from 28 to 107, with an average of one marker every $1.8 \mathrm{cM}$ and a total map length of $860.1 \mathrm{cM}$ (Figure 2B; Table 1; Supplementary Table 2). The SC locus mapped to a single QTL on LG 5 with a maximum LOD value of 7.17 explaining $38.4 \%$ of the phenotypic variance (Figure 2C). An effect plot for the most significant marker, m1042, determined the contribution of the allelic states (A, $\mathrm{H}$, and $\mathrm{B}$ ) to the respective phenotypes (Figure $2 \mathrm{D}$ ). In the homozygous state for the allele contributed by the SC parent (AA), m1042 was associated with an increase of $>40 \%$ in selfcompatible pollen in an in vitro self-pollination assay. The QTL found coincided with the QTL reported in Do Canto et al. (2018) as confirmed by the close physical proximity of m1042 and the two flanking markers, reported by Do Canto et al. (2018), on the Italian ryegrass reference assembly (data not shown).

\section{Segregation Distortion Analysis}

Analysis of segregation distortion performed using the genetic map employed for QTL mapping identified a region on LG 5 deviating from the 1:2:1 marker segregation expected in an $\mathrm{F}_{2}$ population. The region showed a 1:1 segregation in two genotypic classes $(\mathrm{AA}$ and $\mathrm{AB})$ and overlapped with the region harboring the significant QTL for SC (Figure 2C). Similarly, four co-segregating markers (m1609, m113, m1780, and $\mathrm{m} 1440)$ at the end of LG 2 showed significantly distorted segregation.

\section{Fine Mapping and Identification of Candidate Genes}

To further dissect the SC locus, a total of 2,056 $\mathrm{F}_{2}$ plants were genotyped with the two markers, G05_065 and G06_096, known to flank the locus in Do Canto et al. (2018) (Supplementary Table 3). Thirty plants with recombination events between G05_065 and G06_096 were identified, nine of which survived and were further genotyped with the 23 newly developed markers (Supplementary Table 4). These additional genotyping data were used to generate a local linkage map and to 
TABLE 1 | Descriptive statistics of the genotyping-by-sequencing-based genetic linkage map developed from $68 F_{2}$ individuals of perennial ryegrass segregating for self-compatibility.

\begin{tabular}{|c|c|c|c|c|}
\hline Linkage group & Number of markers & Length (cM) & Average spacing (cM) & Maximum spacing (cM) \\
\hline 1 & 28 & 44.7 & 1.7 & 5.3 \\
\hline 2 & 89 & 135.3 & 1.5 & 11.8 \\
\hline 3 & 79 & 183.3 & 2.3 & 22.7 \\
\hline 4 & 107 & 237.8 & 2.2 & 44.2 \\
\hline 5 & 43 & 50 & 1.2 & 7.5 \\
\hline 6 & 52 & 85 & 1.7 & 15.9 \\
\hline 7 & 75 & 124 & 1.7 & 11.5 \\
\hline Overall & 473 & 860.1 & 1.8 & 44.2 \\
\hline
\end{tabular}

Length, average and maximum spacing for each linkage group are indicated in centimorgan (cM).

estimate the genetic distances between those markers (Figure 3). The locus delimited by G05_065 and G06_096 spanned 1.44cM, comparable to the genetic distance $(1.6 \mathrm{cM})$ found by Do Canto et al. (2016).

Of the nine recombinants utilized for the calculation of genetic distances, five flowered and underwent in vitro pollinations, narrowing the region determining SC to $0.26 \mathrm{cM}$ between the markers SF28 and SF61. The position of the fine mapped locus was supported by recombination in the interval between SF3 and SF28 found in P5E10 and by one individual with recombination delimited by marker SF61 in P11C9 (Figure 3). Within the narrower region, one additional plant $(\mathrm{P} 20 \mathrm{H} 12)$ carried recombination between SF3 and SF97, dividing the SC locus into two regions of 0.09 and $0.17 \mathrm{cM}$. However, $\mathrm{P} 20 \mathrm{H} 12$ did not produce viable pollen, preventing phenotyping and the assignment of the SC co-segregating locus into one of the two subregions.

SF28 and SF61 mapped on two consecutive scaffolds of the Italian ryegrass genome assembly. This orthologous region spanned $\sim 3 \mathrm{Mb}$ and contained at least one sequence gap. In the interval, 57 protein-coding genes were predicted (Supplementary Table 5). The marker SF28 overlapped with Lmu01_3856G0000030, while SF61 mapped close to Lmu01_702G0000540, the most proximal gene inside the SC locus. The alignment of its proteome to the perennial ryegrass gene model revealed the presence of 27 highly similar genes distributed on 17 scaffolds. In barley, the region orthologous to the locus co-segregating with $\mathrm{SC}$ was $\sim 3.6 \mathrm{Mb}$ in size and contained 27 genes with HORVU5Hr1G023880 and HORVU5Hr1G024410 limiting the region on the SF28 and SF61 sides, respectively. Fourteen genes had hits to the Italian ryegrass genes of the SC locus (Supplementary Table 5). The orthologous region in rice was located between LOC_Os12g36760 and LOC_Os12g35670 on chromosome 12 and contained 76 genes, 16 of which had similarity to the genes predicted in Italian ryegrass. These orthologous regions from four different species allowed the compilation of a set of candidate genes based on their annotation, described function, and information on organ-specific expression (Table 2).

\section{DISCUSSION}

The identification of loci non-allelic to $S$ and $Z$ suggests that the grass SI system relies on a network of genes needed for the recognition, signal transmission, and rejection or acceptance of self-pollen. How these loci interact with each other and their function in the SI downstream signaling cascade is yet to be determined. However, the SC locus studied here acts epistatically over $S$ and $Z$ : a pollen grain carrying the SC allele overcomes SI regardless of the composition at $S$ and $Z$ and induces a 1:1 segregation in two SC classes: $50 \%$ and $100 \%$ SC (AriasAguirre et al., 2013; Do Canto et al., 2018). Genetically, this segregation pattern is only explained by the action of a single gametophytically-acting gene. Under such a hypothesis, only the heterozygote and homozygote for the SC allele are transmitted to the $\mathrm{F}_{2}$ generation at a $1: 1$ ratio, resulting in the $50 \%$ and $100 \%$ pollen compatibility phenotypes. In our study, we observed the 1:1 phenotypic segregation pattern and surveyed a newly developed genome-wide linkage map. We found that the single QTL on LG 5 was not interacting with other genomic regions, confirming the single-locus inheritance.

Additional evidence was provided by the presence of a major region with distorted segregation in correspondence of the QTL on LG 5. In plants with a functional SI system, the segregation of an SC gene in certain crosses can lead to segregation distortion (Thorogood et al., 2005). In our population, male gametes in the $\mathrm{F}_{1}$ plants carrying the non-SC allele were not transmitted to the $F_{2}$ generation, causing distorted segregation of markers linked to the SC causal gene. This has also been demonstrated recently by Slatter et al. (2020), where the QTL underlying the SC locus on LG 6 in perennial ryegrass overlapped with maximum marker segregation distortion. This has relevant implications for future studies aimed at discovering novel SC sources, as the identification of distorted regions can be used as a strategy to map their position in the genome. Mapping on the basis of segregation distortion represents a valid alternative to the lowthroughput phenotyping provided by in vitro pollinations assays, and it is not dependent on the duration of the flowering period. In addition, as no phenotyping is needed, it allows the screening of larger populations resulting in higher mapping resolution. A less relevant, yet significantly distorted region was also present on 


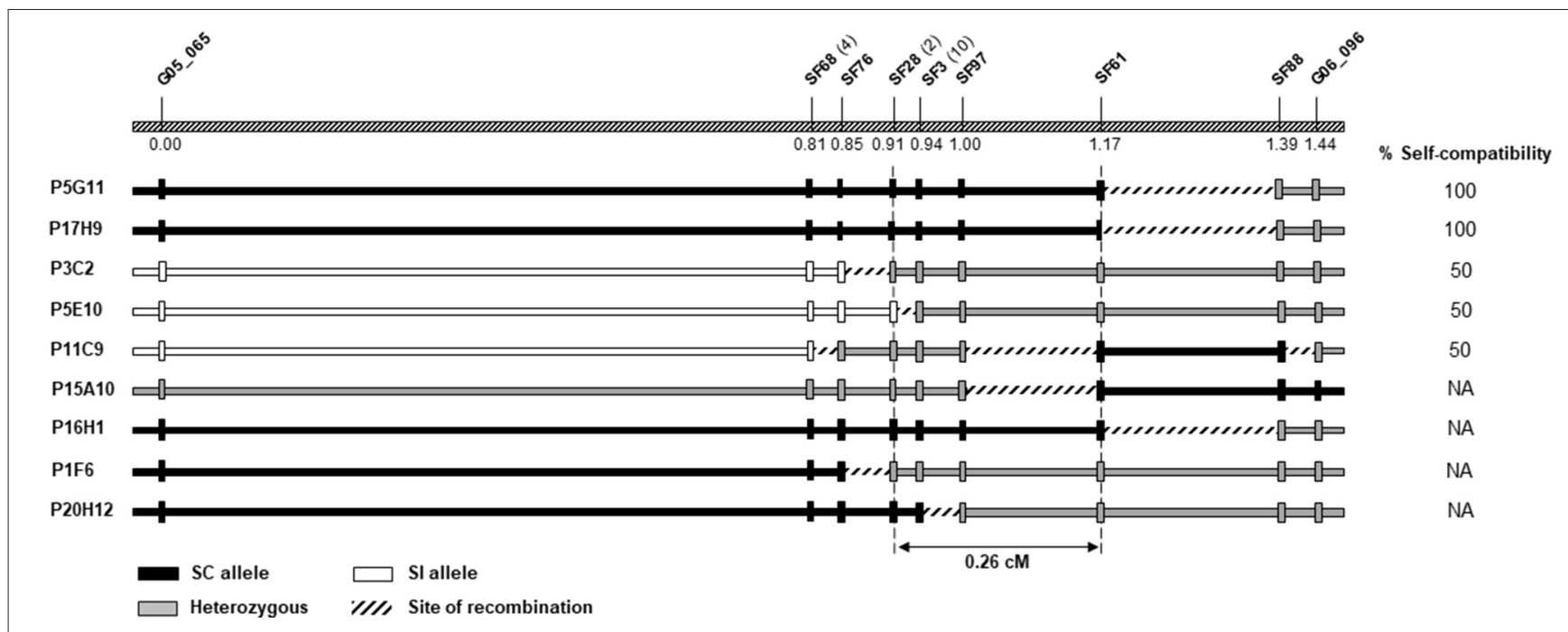

FIGURE 3 | Fine mapping of the self-compatibility (SC) locus on linkage group 5. The top bar shows the genetic order and distances in centimorgan (cM) of the nine informative markers used for the calculation of genetic distances. For SF68, SF28, and SF3, the number of co-segregating markers is indicated in brackets. Genotypes at each marker (vertical bars) and haplotype configuration (horizontal bars) for the nine individuals screened with G05_065 and G06_096 and the newly developed markers are shown. The column on the right side reports the phenotypes, when available. The dashed vertical lines at SF28 and SF61 limit the SC locus.

LG 2. There are several described biological reasons underlying marker segregation distortion (Xu et al., 1997), thus it is difficult to identify its cause in this case.

After low-resolution QTL mapping, fine mapping resulted in the allocation of the locus to a $0.26-\mathrm{cM}$ region between the markers SF28 and SF61, significantly reducing the extent of the previously described locus (Do Canto et al., 2018) to a sub-cM region spanning $\sim 3 \mathrm{Mb}$ on the Italian ryegrass reference assembly. Importantly, there were nine additional markers that co-segregated with SF28, SF97, and SF61 due to the lack of recombination events in the nine individuals that we identified. Such markers will be a valuable resource to further dissect genetically the SC locus, should additional $\mathrm{F}_{2}$ individuals be screened.

In the absence of a contiguous reference genome for perennial ryegrass, we cannot precisely define the genes present in the interval co-segregating with SC. However, given the high genome synteny with Italian ryegrass, barley, and rice, it is relevant to assess and compare the genes annotated in the orthologous interval of their reference genomes as a proxy to suggesting candidate genes. On the Italian ryegrass reference assembly, the interval contains 57 protein-coding genes and we foresee to prioritize some of them as candidates responsible for SC based on their predicted function. The region showed conservation compared to other grasses, spanning a similar physical size on barley chromosome 5 and containing 27 genes. In rice, the SC locus ortholog was on chromosome 12. The wealth of functional information available for rice, barley, and Arabidopsis thaliana genes and similarity to other plant genes with known function allowed prioritization of genes for future identification of the SC determinant in perennial ryegrass.
Preliminary studies on the molecular determination of SI and SC suggested that $\mathrm{Ca}^{2+}$ signaling is involved in the recognition and/or inhibition of incompatible/compatible pollen. In fact, two independent expression studies registered an enrichment of transcripts during the SI response predicted to code for proteins containing calcium-binding domains in perennial ryegrass and sheepgrass (Leymus chinensis Trin.) (Yang et al., 2009; Chen et al., 2019). Two genes in the fine mapped SC locus region (Lmu01_3856G0000420 and Lmu01_3856G0000430) encode for proteins with calcium-dependent functions. Their orthologs in Arabidopsis thaliana, At2G18750, and At1G09170, encode for a calmodulin-binding protein and a kinesin motor protein with a calponin (calcium-binding protein) domain, respectively. No At2G18750 expression in floral tissues has been described so far. However, At1G09170 is co-expressed in mature pollen with several genes involved in mediating microtubule organization during pollen tube polar growth (Schneider and Persson, 2015). Interestingly, their orthologs in perennial ryegrass were reported to be upregulated during compatible reactions (Byrne et al., 2015), making them strong candidates for causing SC in our population.

Another relevant candidate gene is Lmu01_3856G0000440, whose function was annotated as a leucine-rich repeat extensin (LRX). Its ortholog in Arabidopsis thaliana (At2G15880) shows pollen-specific expression, and the rice ortholog (LOC_Os12g356710) shows high expression during pollen germination. LRXs are cell wall proteins and part of a signaling system that transfers extracellular signals from the cell wall to the cytoplasm to regulate cell growth in vegetative tissue and in the pollen tube (Herger et al., 2019). In several recent studies in Arabidopsis thaliana, lrx mutants show severe defects in 
TABLE 2 | List of the seven prioritized candidate genes co-segregating with self-compatibility, their annotation, and orthologs in perennial ryegrass (Lolium perenne L.), barley (Hordeum vulgare L.), rice (Oryza sativa L.), and Arabidopsis thaliana.

\begin{tabular}{|c|c|c|c|c|c|}
\hline Italian ryegrass & Perennial ryegrass & Barley & Rice & Arabidopsis thaliana & Functional annotation \\
\hline Lmu01_3856G0000060 & $\begin{array}{l}\text { maker- } \\
\text { scaffold_1442|refo021156- } \\
\text { exonerate_est2genome- } \\
\text { gene-0.1-mRNA-1 }\end{array}$ & - & - & - & $\begin{array}{l}\text { Protein of unknown function } \\
\text { (DUF295), F-box-like protein }\end{array}$ \\
\hline Lmu01_3856G0000100 & $\begin{array}{l}\text { maker- } \\
\text { scaffold_10104|ref00 } \\
\text { 10435- } \\
\text { exonerate_est2genome- } \\
\text { gene-0.3-mRNA-1 }\end{array}$ & $\begin{array}{l}\text { HORVU5Hr1G } \\
024060.1\end{array}$ & LOC_Os12g36670.1 & AT5G27920 & F-box family protein \\
\hline Lmu01_3856G0000370 & $\begin{array}{l}\text { maker- } \\
\text { scaffold_2321|ref0036192- } \\
\text { exonerate_est2genome- } \\
\text { gene-0.1-mRNA-1 }\end{array}$ & - & - & AT1G13570 & $\begin{array}{l}\text { F-box/RNI-like superfamily } \\
\text { protein }\end{array}$ \\
\hline Lmu01_3856G0000420 & $\begin{array}{l}\text { maker- } \\
\text { scaffold_792|ref0008797- } \\
\text { exonerate_est2genome- } \\
\text { gene-0.1-mRNA-1 }\end{array}$ & $\begin{array}{l}\text { HORVU5Hr1GO } \\
24400.1\end{array}$ & - & AT2G18750 & Calmodulin-binding protein \\
\hline Lmu01_3856G0000430 & $\begin{array}{l}\text { maker- } \\
\text { scaffold_792|ref0008797- } \\
\text { exonerate_est2genome- } \\
\text { gene-0.2-mRNA-1 }\end{array}$ & $\begin{array}{l}\text { HORVU5Hr1 } \\
\text { G024390.9 }\end{array}$ & LOC_Os12g36100.1 & AT1G09170 & $\begin{array}{l}\text { P-loop nucleoside triphosphate } \\
\text { hydrolases superfamily protein } \\
\text { with Calponin Homology } \\
\text { domain }\end{array}$ \\
\hline Lmu01_3856G0000440 & - & $\begin{array}{l}\text { HORVU5Hr1 } \\
\text { G024380.4 }\end{array}$ & LOC_Os12g35710.1 & AT2G15880 & $\begin{array}{l}\text { Leucine-rich repeat extensin } \\
(\mathrm{LRX})\end{array}$ \\
\hline Lmu01_702G0000440 & - & $\begin{array}{l}\text { HORVU5Hr1 } \\
\text { G024410 }\end{array}$ & LOC_Os12g35670 & AT4G15800 & $\begin{array}{l}\text { Rapid alkalinization Factor } \\
\text { (RALF) protein }\end{array}$ \\
\hline
\end{tabular}

pollen germination and pollen tube growth (Fabrice et al., 2018; Sede et al., 2018; Wang et al., 2018). However, manipulating $\mathrm{Ca}^{2+}$ availability alleviates these defects, suggesting that LRX proteins can regulate $\mathrm{Ca}^{2+}$-related processes. In carrying out their regulatory function, LRX proteins interact with short cysteine-rich peptides known as rapid alkalinization factors (RALFs). Interestingly, a gene encoding a RALF-like protein (Lmu01_702G0000440) is also present in the SC co-segregating region. RALFs induce a rapid alkalinization of extracellular space by increasing the cytoplasmic $\mathrm{Ca}^{2+}$ concentration that leads to calcium-dependent signaling events. They control several plant stress response mechanisms, growth, and development (Murphy and De Smet, 2014), and pollen-specific RALFs regulating pollen tube growth are described in tomato (Solanum lycopersicum L.) (Covey et al., 2010) and Arabidopsis thaliana (Mecchia et al., 2017; Moussu et al., 2020). Both LRX- and RALF-like-encoding genes are strong candidates for causing the inhibition of selfpollen tubes. They could be a fundamental component of the process that inhibits self-pollen tubes by transducing SI signals to activate the downstream pathway that modulates the $\mathrm{Ca}^{2+}$ gradient necessary for pollen tube growth. A disruptive mutation in such genes could interrupt the inhibitory mechanism, leading to SC.

Three other genes located in the narrower interval encode for F-box proteins (Lmu01_3856G0000060, Lmu01_3856G0000100, and Lmu01_3856G0000370). F-box genes constitute one of the largest gene families in plants, involved in the degradation of cellular proteins (Lechner et al., 2006). F-box proteins form a subunit of the Skp1-cullin-F-box (SCF) complex that confers specificity for a target substrate to be degraded and are involved in important biological processes such as embryogenesis, floral development, plant growth and development, biotic and abiotic stress, hormonal responses, and senescence, among others (Gupta et al., 2015). S-locus F-box genes (SFB or SLF, depending on the family) are known as the male determinants of $S$ RNase-based gametophytic SI systems (i.e., Rosaceae, Solanaceae, Scrophulariaceae, and Rubiaceae). In these systems, the female determinant encodes for transmembrane $S$-RNase in the stigma with high cytotoxic activity for the pollen tube. In a non-selfpollination event, SFBs identify and degrade non-self $S$-RNases allowing fertilization to occur unimpeded via the ubiquitin-26S proteasome pathway. Whereas in self-pollination, self $S$-RNases evade degradation and exert cytotoxicity inside the pollen tube to inhibit its growth (Hua and Kao, 2008; McClure et al., 2011). A neo-functionalization of an F-box gene was reported in two recent studies to underly the Sli locus, responsible for breaking down the stylar incompatible response in potato (Solanum tuberosum L.) (Eggers et al., 2021; Ma et al., 2021). Similarly, an F-box gene represents an interesting candidate with a putative role in the complex downstream pathway that leads to rejection of self-pollen in grasses and a disruptive mutation might lead to SC by halting its inhibiting function.

The genes discussed here are plausible candidates to cause SC, selected on the basis of their annotation and function described in previous studies. However, it is not possible to exclude the remaining genes in the genomic region co-segregating with SC as potential candidates. Only additional, complementary analyses will allow the identification and confirmation of the molecular determinant of SC. Among these, targeted sequencing and sequence comparison of such genes in our SC population 
and diverse SI genotypes can contribute to reducing the number of candidate genes. Furthermore, techniques, such as Targeting Induced Local Lesions in Genomes (TILLING), offer opportunities to screen for point mutations in candidate genes in populations mutagenized by chemical treatment and evaluate if those lead to an SC phenotype (Manzanares et al., 2016b). Also, recent advances in genome editing through CRISPR/Cas9 and its implementation in ryegrass (Zhang et al., 2020) makes this an attractive alternative to achieve targeted gene inactivation of SC candidate genes to unequivocally determine their function.

\section{CONCLUSIONS}

In this study, we provide additional evidence of a major QTL on LG 5 being solely responsible for SC in a perennial ryegrass population and demonstrate that segregation distortion can be an efficient strategy to identify loci conferring SC. Additionally, we developed new markers and fine mapped the previously described region from $1.6 \mathrm{cM}$ down to $0.26 \mathrm{cM}$. Genome synteny to other grass species was used to determine a $3-\mathrm{Mb}$ orthologous region in Italian ryegrass and to find the gene content in the region co-segregating with SC. From a total of 57 genes, seven encode for functionally relevant proteins and will be prioritized for validation as genetic determinants of SC. Furthermore, the genetic markers developed for fine mapping are tightly linked to the SC gene and can readily be converted into high throughput genotyping assays. They provide a convenient molecular tool to transfer SC to elite germplasm for the development of inbred lines-based breeding strategies.

\section{DATA AVAILABILITY STATEMENT}

The original contributions presented in the study are publicly available. This data can be found here: NCBI repository, accession number: PRJNA724991.

\section{REFERENCES}

Arias-Aguirre, A., Studer, B., Do Canto, J., Frei, U., and Lübberstedt, T. (2013). Mapping a new source of self-fertility in perennial ryegrass (Lolium perenne L.). Plant Breed Biotechnol. 1, 385-395. doi: 10.9787/PBB.2013.1.4.385

Begheyn, R. F., Yates, S. A., Sykes, T., and Studer, B. (2018). Genetic Loci Governing androgenic capacity in perennial ryegrass (Lolium perenne L.). G3 Genes, Genomes, Genet 8, 1897-1908. doi: 10.1534/g3.117.300550

Bian, X. Y., Friedrich, A., Bai, J. R., Baumann, U., Hayman, D. L., and Barker, S. J., et al. (2004). High-resolution mapping of the $\mathrm{S}$ and $\mathrm{Z}$ loci of Phalaris coerulescens. Genome 47, 918-930. doi: 10.1139/g04-017

Broman, K. W., Wu, H., Sen, S., and Churchill, G. A. (2003). R/qtl: QTL mapping in experimental crosses. Bioinformatics 19, 889-890. doi: 10.1093/bioinformatics/btg112

Byrne, S. L., Nagy, I., Pfeifer, M., Armstead, I., Swain, S., and Studer, B., et al. (2015). A synteny-based draft genome sequence of the forage grass Lolium perenne. Plant J. 84, 816-826. doi: 10.1111/tpj.13037

Camacho, C., Coulouris, G., Avagyan, V., Ma, N., Papadopoulos, J., and Bealer, K., et al. (2009). BLAST+: Architecture and applications. BMC Bioinform. 10, 1-9. doi: $10.1186 / 1471-2105-10-421$

\section{AUTHOR CONTRIBUTIONS}

CC, CM, MK, TL, and BS: study conception. JD and TL: generation of plant material. CC, CM, and SY: GBS library preparation, sequencing, and bioinformatics. CC: genetic map development, QTL mapping, and analysis of segregation distortion. CC, CM, SY, and DC: marker development, genotyping, and marker data analysis. CC and DC: comparative genomic analysis. $\mathrm{MK}$ and BS: funding acquisition. CC, CM, DC, and BS: manuscript writing. All authors read and approved the final version of the manuscript.

\section{FUNDING}

This work was supported by the European Union's Horizon 2020 Research and Innovation Programme Marie SkłodowskaCurie grant agreement no 722338 - PlantHUB and by the Swiss National Science Foundation (grant number 310030_197708).

\section{ACKNOWLEDGMENTS}

The authors wish to acknowledge Ingrid Stoffel-Studer and Dr. Timothy Sykes from the Molecular Plant Breeding group at ETH Zurich for assisting in DNA extraction and GBS library preparation, the FCGZ for sequencing service, the Zurich-Basel Plant Science Center for professional management of the PlantHUB programme, and Nic Boerboom from DSV Zaden Nederland B.V for the helpful comments on the manuscript.

\section{SUPPLEMENTARY MATERIAL}

The Supplementary Material for this article can be found online at: https://www.frontiersin.org/articles/10.3389/fpls.2021. 707901/full\#supplementary-material

Capstaff, N. M., and Miller, A. J. (2018). Improving the yield and nutritional quality of forage crops. Front. Plant Sci. 9, 1-18. doi: 10.3389/fpls.2018. 00535

Chen, S., Jia, J., Cheng, L., Zhao, P., Qi, D., and Yang, W., et al. (2019). Transcriptomic analysis reveals a comprehensive calcium- and phytohormonedominated signaling response in Leymus chinensis self-incompatibility. Int. J. Mol. Sci. 20, 1-24. doi: 10.3390/ijms20092356

Copetti, D., Yates, S. A., Vogt, M. M., Russo, G., Grieder, C., and Kölliker, R., et al. (2021). Evidence for high intergenic sequence variation in heterozygous Italian ryegrass (Lolium multiflorum Lam.) genome revealed by a highquality draft diploid genome assembly. bioRxiv. doi: 10.1101/2021.05.05. 442707

Cornish, M. A., Hayward, M. D., and Lawrence, M. J. (1979). Self-incompatibility in ryegrass. Heredity 43, 95-106. doi: 10.1038/hdy.1979.63

Covey, P. A., Subbaiah, C. C., and Parsons, R. L., et al. (2010). A pollen-specific RALF from tomato that regulates pollen tube elongation. Plant Physiol. 153, 703-715. doi: 10.1104/pp.110.155457

Do Canto, J., Studer, B., Frei, U., and Lübberstedt, T. (2018). Fine mapping a self-fertility locus in perennial ryegrass. Theor. Appl. Genet. 131, 817-827. doi: $10.1007 /$ s00122-017-3038-6 
Do Canto, J., Studer, B., and Lübberstedt, T. (2016). Overcoming selfincompatibility in grasses: a pathway to hybrid breeding. Theor. Appl. Genet. 129, 1815-1829. doi: 10.1007/s00122-016-2775-2

Eggers, E. J., van der Burgt, A., van Heusde, S. A., de Vries, M. E., Visser, R. G., and Bachem, C. W., et al. (2021). Neofunctionalisation of the Sli gene leads to self-compatibility and facilitates precision breeding in potato. Nat. Commun. 12, 1-9. doi: 10.1038/s41467-021-24267-6

Egorova, I. A., Peneva, T. I., Baranova, O. A., and Voylokov, A. V. (2000). Analysis of linkage between biochemical and morphological markers of rye chromosomes $1 \mathrm{R}, 2 \mathrm{R}$, and $5 \mathrm{R}$ and mutations of selffertility at the main incompatibility loci. Russ. J. Genet. 36, 1423-1430. doi: 10.1023/A:1009075408921

Fabrice, T. N., Vogler, H., Draeger, C., Munglani, G., Gupta, S., and Herger, A. G., et al. (2018). LRX proteins play a crucial role in pollen grain and pollen tube cell wall development. Plant Physiol. 176, 1981-1992. doi: 10.1104/pp.17.01374

Gupta, S., Garg, V., Kant, C., and Bhatia, S. (2015). Genome-wide survey and expression analysis of F-box genes in chickpea. BMC Genomics 16, 1-15. doi: 10.1186/s12864-015-1293-y

Hackauf, B., and Wehling, P. (2005). Approaching the self-incompatibility locus $\mathrm{Z}$ in rye (Secale cereale L.) via comparative genetics. Theor. Appl. Genet. 110, 832-845. doi: 10.1007/s00122-004-1869-4

Hayman, D. (1956). The Genetical Control of Incompatibility in Phalaris coerulescens Desf. Aust. J. Biol. Sci. 9:321. doi: 10.1071/BI9560321

Herger, A., Dünser, K., Kleine-Vehn, J., and Ringli, C. (2019). Leucine-rich repeat extensin proteins and their role in cell wall sensing. Curr. Biol. 29, R851-R858. doi: 10.1016/j.cub.2019.07.039

Herridge, R. P., Macknight, R. C., and Brownfield, L. R. (2020). Prospects for F1 hybrid production in ryegrass. New Zeal. J. Agric. Res. 63, 405-415. doi: 10.1080/00288233.2018.155 9867

Hua, Z., and Kao, T. H. (2008). Identification of major lysine residues of S3-RNase of Petunia inflata involved in ubiquitin-26S proteasome-mediated degradation in vitro. Plant J. 54, 1094-1104. doi: 10.1111/j.1365-313X.2008.03487.x

Jansky, S. H., Charkowski, A. O., Douches, D. S., Gusmini, G., Richael, C., and Bethke, P. C., et al. (2016). Reinventing potato as a diploid inbred line-based crop. Crop Sci. 56, 1412-1422. doi: 10.2135/cropsci2015.12.0740

Jensen, L. B., Andersen, J. R., Frei, U., Xing, Y., Taylor, C., and Bach, P., et al. (2005). QTL mapping of vernalization response in perennial ryegrass (Lolium perenne L.) reveals co-location with an orthologue of wheat VRN1. Theor. Appl. Genet. 110, 527-536. doi: 10.1007/s00122-004-1865-8

Kakeda, K., Ibuki, T., Suzuki, J., Tadano, H., Kurita, Y., and Hanai, Y., et al. (2008). Molecular and genetic characterization of the S locus in Hordeum bulbosum L., a wild self-incompatible species related to cultivated barley. Mol. Genet. Genom. 280, 509-519. doi: 10.1007/s00438-008-0383-9

Klaas, M., Yang, B., Bosch, M., Thorogood, D., Manzanares, C., and Armstead, I. P., et al. (2011). Progress towards elucidating the mechanisms of selfincompatibility in the grasses: Further insights from studies in Lolium. Ann. Bot. 108, 677-685. doi: 10.1093/aob/mcr186

Kosambi, D. D. (1943). The estimation of map distances from recombination values. Ann. Hum. Genet. 12, 172-175. doi: 10.1111/j.1469-1809.1943.tb02321.x

Laidig, F., Piepho, H. P., Drobek, T., and Meyer, U. (2014). Genetic and nongenetic long-term trends of 12 different crops in German official variety performance trials and on-farm yield trends. Theor. Appl. Genet. 127, 2599-2617. doi: 10.1007/s00122-014-2402-Z

Langmead, B., and Salzberg, S. L. (2012). Fast gapped-read alignment with Bowtie 2. Nat. Methods 9, 357-359. doi: 10.1038/nmeth.1923

Lechner, E., Achard, P., Vansiri, A., Potuschak, T., and Genschik, P. (2006). F-box proteins everywhere. Curr. Opin. Plant Biol. 9, 631-638. doi: 10.1016/j.pbi.2006.09.003

Li, H., Handsaker, B., Wysoker, A., Fennell, T., Ruan, J., and Homer, N., et al. (2009). The Sequence Alignment/Map format and SAMtools. Bioinformatics 25, 2078-2079. doi: 10.1093/bioinformatics/btp352

Lundqvist, A. (1956). Self-incompatibility in rye. I. Genetic control in the diploid. Hereditas 44, 193-256. doi: 10.1111/j.1601-5223.1956.tb03021.x

Ma, L., Zhang, C., Zhang, B., Tang, F., Li, F., and Liao, Q., et al. (2021). A nonSlocus F-box gene breaks self-incompatibility in diploid potatoes. Nat. Commun. 12, 1-8. doi: 10.1038/s41467-021-24266-7

Manzanares, C., Barth, S., Thorogood, D., Byrne, S. L., Yates, S. A., and Czaban, A., et al. (2016a). A gene encoding a DUF247 domain protein cosegregates with the
S self-incompatibility locus in perennial ryegrass. Mol. Biol. Evol. 33, 870-884. doi: $10.1093 / \mathrm{molbev} / \mathrm{msv} 335$

Manzanares, C., Yates, S., Ruckle, M., Nay, M., and Studer, B. (2016b). TILLING in forage grasses for gene discovery and breeding improvement. N. Biotechnol. 33, 594-603. doi: 10.1016/j.nbt.2016.02.009

Margarido, G. R. A., Souza, A. P., and Garcia, A. A. F. (2007). OneMap: Software for genetic mapping in outcrossing species. Hereditas 144, 78-79. doi: 10.1111/j.2007.0018-0661.02000.x

Martin, F. W. (1959). Staining and observing pollen tubes in the style by means of fluorescence. Biotech. Histochem. 34, 125-128. doi: $10.3109 / 10520295909114663$

Mascher, M., Gundlach, H., Himmelbach, A., Beier, S., Twardziok, S. O., and Wicker, T., et al. (2017). A chromosome conformation capture ordered sequence of the barley genome. Nature 544, 427-433. doi: 10.1038/nature22043

McClure, B., Cruz-García, F., and Romero, C. (2011). Compatibility and incompatibility in S-RNase-based systems. Ann. Bot. 108, 647-658. doi: $10.1093 / \mathrm{aob} / \mathrm{mcr} 179$

Mecchia, M. A., Santos-Fernandez, G., Duss, N. N., Somoza, S. C., BoissonDernier, A., and Gagliardini, V., et al. (2017). RALF4/19 peptides interact with LRX proteins to control pollen tube growth in Arabidopsis. Science 358, 1600-1603. doi: 10.1126/science.aao5467

Moussu, S., Broyart, C., Santos-Fernandez, G., Augustin, S., Wehrle, S., and Grossniklaus, U., et al. (2020). Structural basis for recognition of RALF peptides by LRX proteins during pollen tube growth. Proc. Natl. Acad. Sci. 117, 7494-7503. doi: 10.1073/pnas.2000100117

Murphy, E., and De Smet, I. (2014). Understanding the RALF family: A tale of many species. Trends Plant Sci. 19, 664-671. doi: 10.1016/j.tplants.2014.06.005

Ouyang, S., Zhu, W., Hamilton, J., Lin, H., Campbell, M., and Childs, K., et al. (2007). The TIGR rice genome annotation resource: improvements and new features. Nucleic Acids Res. 35, 8-11. doi: 10.1093/nar/gkl.976

R Core Team (2020). R: a language and environment for statistical computing. $\mathrm{R}$ Foundation for Statistical Computing, Vienna, Austria. Available online at: https://www.R-project.org/.

Robinson, J. T., Thorvaldsdóttir, H., Winckler, W., Guttman, M., Lander, E. S., and Getz, G., et al. (2011). Integrative genomics viewer. Nat. Biotechnol. 29, 24-26. doi: $10.1038 /$ nbt.1754

Schneider, R., and Persson, S. (2015). Connecting two arrays: the emerging role of actin-microtubule cross-linking motor proteins. Front. Plant Sci. 6, 1-7. doi: $10.3389 /$ fpls. 2015.00415

Sede, A. R., Borassi, C., Wengier, D. L., Mecchia, M. A., Estevez, J. M., and Muschietti, J. P. (2018). Arabidopsis pollen extensins LRX are required for cell wall integrity during pollen tube growth. FEBS Lett. 592, 233-243. doi: 10.1002/1873-3468.12947

Shinozuka, H., Cogan, N. O. I., Smith, K. F., Spangenberg, G. C., and Forster, J. W. (2010). Fine-scale comparative genetic and physical mapping supports map-based cloning strategies for the self-incompatibility loci of perennial ryegrass (Lolium perenne L.). Plant Mol. Biol. 72, 343-355. doi: 10.1007/s11103-009-9574-y

Slatter, L. M., Barth, S., Manzanares, C., Velmurugan, J., Place, I., and Thorogood, D. (2020). A new genetic locus for self-compatibility in the outcrossing grass species perennial ryegrass (Lolium perenne). Ann. Bot. 127, 1-8. doi: $10.1093 / \mathrm{aob} / \mathrm{mcaa} 140$

Studer, B., Asp, T., Frei, U., Hentrup, S., Meally, H., and Guillard, A., et al. (2008). Expressed sequence tag-derived microsatellite markers in perennial ryegrass (Lolium perenne L.). Mol. Breed. 21, 533-548. doi: 10.1007/s11032-007-9148-0

The Arabidopsis Genome Initiative (2000). Analysis of the genome sequence of the flowering plant Arabidopsis thaliana. Nature 408, 796-815. doi: $10.1038 / 35048692$

Thorogood, D., and Armstead, I. (2002). Self-incompatibility in ryegrass 12. Genotyping and mapping the $\mathrm{S}$ and $\mathrm{Z}$ loci of Lolium perenne L. Heredity 71, 385-390. doi: 10.1038/sj.hdy.6800071

Thorogood, D., Armstead, I. P., Turner, L. B., Humphreys, M. O., and Hayward, M. D. (2005). Identification and mode of action of self-compatibility loci in Lolium perenne L. Heredity 94, 356-363. doi: 10.1038/sj.hdy. 6800582

Voylokov, A. V., Fuong, F. T., and Smirnov, V. G. (1993). Genetic studies of selffertility in rye (Secale cereale L.). 1. The identification of genotypes of self-fertile lines for the Sf alleles of self-incompatibility genes. Theor. Appl. Genet. 87, 616-618. doi: 10.1007/BF00221887 
Voylokov, A. V., Korzun, V., and Börner, A. (1998). Mapping of three self-fertility mutations in rye (Secale cereale L.) using RFLP, isozyme and morphological markers. Theor. Appl. Genet. 97, 147-153. doi: 10.1007/s001220050879

Wang, X., Wang, K., Yin, G., Liu, X., Liu, M., and Cao, N., et al. (2018). Pollenexpressed leucine-rich repeat extensins are essential for pollen germination and growth. Plant Physiol. 176, 1993-2006. doi: 10.1104/pp.17.01241

Wang, Y., Tang, H., Debarry, J. D., Tan, X., Li, J., and Wang, X., et al. (2012). MCScanX: A toolkit for detection and evolutionary analysis of gene synteny and collinearity. Nucleic Acids Res. 40, 1-14. doi: 10.1093/nar/gkr1293

Xu, Y., Zhu, L., Xiao, J., Huang, N., and McCouch, S. R. (1997). Chromosomal regions associated with segregation distortion of molecular markers in F2, backcross, doubled haploid, and recombinant inbred populations in rice (Oryza sativa L.). Mol. Gen. Genet. 253, 535-545. doi: 10.1007/s004380050355

Yang, B., Thorogood, D., Armstead, I. P., Franklin, F. C. H., and Barth, S. (2009). Identification of genes expressed during the self-incompatibility response in perennial ryegrass (Lolium perenne L.). Plant Mol. Biol. 70, 709-723. doi: 10.1007/s11103-009-9501-2

Yates, S. A., and Studer, B. (2021). GBSmode: a pipeline for haplotype-aware analysis of genotyping-by-sequencing data. bioRxiv. doi: 10.1101/2021.09.20.461130

Ye, J., Coulouris, G., Zaretskaya, I., Cutcutache, I., Rozen, S., and Madden, T. L. (2012). Primer-BLAST: a tool to design target-specific primers for polymerase chain reaction. BMC Bioinform. 13:134. doi: 10.1186/1471-2105-13-134

Young, N. D., and Tanksley, S. D. (1989). Restriction fragment length polymorphism maps and the concept of graphical genotypes. Theor. Appl. Genet. 77, 95-101. doi: 10.1007/BF00292322
Zhang, Y., Ran, Y., Nagy, I., Lenk, I., Qiu, J. L., and Asp, T., et al. (2020). Targeted mutagenesis in ryegrass (Lolium spp.) using the CRISPR/Cas9 system. Plant Biotechnol. J. 18, 1854-1856. doi: 10.1111/pbi.13359

Zimmermann, P., Hennig, L., and Gruissem, W. (2005). Geneexpression analysis and network discovery using Genevestigator. Trends Plant Sci. 10, 407-409. doi: 10.1016/j.tplants.2005.0 7.003

Conflict of Interest: The authors declare that the research was conducted in the absence of any commercial or financial relationships that could be construed as a potential conflict of interest.

Publisher's Note: All claims expressed in this article are solely those of the authors and do not necessarily represent those of their affiliated organizations, or those of the publisher, the editors and the reviewers. Any product that may be evaluated in this article, or claim that may be made by its manufacturer, is not guaranteed or endorsed by the publisher.

Copyright (c) 2021 Cropano, Manzanares, Yates, Copetti, Do Canto, Lübberstedt, Koch and Studer. This is an open-access article distributed under the terms of the Creative Commons Attribution License (CC BY). The use, distribution or reproduction in other forums is permitted, provided the original author $(s)$ and the copyright owner(s) are credited and that the original publication in this journal is cited, in accordance with accepted academic practice. No use, distribution or reproduction is permitted which does not comply with these terms. 